\title{
FINAL EVOLUTION AND DELAYED EXPLOSIONS OF SPINNING WHITE DWARFS IN SINGLE DEGENERATE MODELS FOR TYPE Ia SUPERNOVAE
}

\author{
Omar G. Benvenuto ${ }^{1,5}$, Jorge A. Panei ${ }^{1,6}$, Ken'ichi Nomoto $^{2,7}$, Hikaru Kitamura ${ }^{3}$, and Izumi Hachisu ${ }^{4}$ \\ ${ }^{1}$ Facultad de Ciencias Astronómicas y Geofísicas, Universidad Nacional de La Plata and Instituto de Astrofísica de La Plata, \\ CCT-CONICET-UNLP. Paseo del Bosque S/N (B1900FWA), La Plata, Argentina; obenvenu@fcaglp.unlp.edu.ar \\ ${ }^{2}$ Kavli Institute for the Physics and Mathematics of the universe (WPI), The University of Tokyo, \\ Kashiwanoha 5-1-5, Kashiwa, Chiba 277-8583, Japan \\ ${ }^{3}$ Department of Physics, Kyoto University, Sakyo-ku, Kyoto 606-8502, Japan \\ ${ }^{4}$ Department of Earth Science and Astronomy, College of Arts and Sciences, The University of Tokyo, \\ Komaba 3-8-1, Meguro-ku, Tokyo 153-8902, Japan \\ ${ }^{5}$ Carrera del Investigador Científico of the Comisión de Investigaciones Científicas (CIC) \\ de la Provincia de Buenos Aires, Buenos Aires, Argentina \\ ${ }^{6}$ Carrera del Investigador Científico y Tecnológico, CONICET, Argentina \\ Received 2015 May 4; accepted 2015 July 15; published 2015 August 5
}

\begin{abstract}
We study the occurrence of delayed SNe Ia in the single degenerate scenario. We assume that a massive carbonoxygen (CO) white dwarf (WD) accretes matter coming from a companion star, making it spin at the critical rate. We assume uniform rotation due to magnetic field coupling. The carbon ignition mass for non-rotating WDs is $M_{\mathrm{ig}}^{\mathrm{NR}} \approx 1.38 M_{\odot}$, while for the case of uniformly rotating WDs it is a few percent larger $\left(M_{\mathrm{ig}}^{\mathrm{R}} \approx 1.43 M_{\odot}\right)$. When accretion rate decreases, the WD begins to lose angular momentum, shrinks, and spins up; however, it does not overflow its critical rotation rate, avoiding mass shedding. Thus, angular momentum losses can lead the CO WD interior to compression and carbon ignition, which would induce an SN Ia. The delay, largely due to the angular momentum losses timescale, may be large enough to allow the companion star to evolve to a He WD, becoming undetectable at the moment of explosion. This scenario supports the occurrence of delayed SNe Ia if the final CO WD mass is $1.38 M_{\odot}<M<1.43 M_{\odot}$. We also find that if the delay is longer than $\sim 3$ Gyr, the WD would become too cold to explode, rather undergoing collapse.
\end{abstract}

Key words: binaries: close - nuclear reactions, nucleosynthesis, abundances - stars: rotation - supernovae: general - white dwarfs

\section{INTRODUCTION}

The origin of Type Ia supernovae ( $\mathrm{SNe}$ Ia) has been one of the central issues of recent astrophysics. The main features of SNe Ia have been firmly accounted for as a consequence of a thermonuclear explosion of a carbon-oxygen $(\mathrm{C}+\mathrm{O})$ white dwarf (WD). Both Chandrasekhar mass and sub-Chandrasekhar mass models have been presented (Hillebrandt \& Niemeyer 2000; Nomoto et al. 2000). However, it is still not clear if the WD accretes $\mathrm{H} / \mathrm{He}$-rich matter from its binary companion (single degenerate (SD) scenario), or two $\mathrm{C}+\mathrm{O}$ WDs merge (double degenerate (DD) scenario; Maoz et al. 2014).

Observations provided some constraints on the nature of companion stars that may be considered as an indication of the occurrence of the SD scenario. These are the presence of circumstellar matter (Patat et al. 2007; Sternberg et al. 2011; Foley et al. 2012) and the detection of hydrogen in the circumstellar-interaction-type SNe (Ia/IIn), such as SN 2002ic (Hamuy et al. 2003) and PTF11kx (Dilday et al. 2012). On the other hand, it has been not possible to detect the presence of companions, e.g., the lack of companion stars in the images of SN 2011fe (Li et al. 2011), some SN Ia remnants (Schaefer \& Pagnotta 2012), SN 1572 (Tycho; Kerzendorf et al. 2009), and SN 1006 (Kerzendorf et al. 2012).

However, the above discussion does not take into account that the accreting WD should be rotating by getting the angular momentum from the accreting materials. Justham (2011) and Di Stefano et al. (2011) presented a spin-down scenario and

\footnotetext{
${ }^{7}$ Hamamatsu Professor.
}

suggested that there should exist a long spin-down phase of the rapidly rotating WD with a timescale of the angular momentum loss ( $J$-loss) from the WD. If so, the donor star in the SD model may exhaust its hydrogen-rich envelope and become a He WD before the SN Ia explosion. Hachisu et al. (2012) and Wang et al. (2014) studied close binary evolutions by taking into account the rotation of accreting WDs and obtained the parameter space (the companion's mass and the binary separation) where the " $J$-loss" evolution actually occurs. Rotating WDs have been studied by Ostriker \& Bodenheimer (1968) and others. More recently, presupernova evolution of rotating WDs has been modeled in 2D by, e.g., Uenishi et al. (2003) and Yoon \& Langer (2005).

In the present study, we have constructed a new 1D stellar evolution code by taking into account rotation. In Section 2, we describe the ingredients of the models as well as the numerical techniques employed. With this code, we have calculated the final evolution of the mass-accreting WDs by assuming that it rotates uniformly because of magnetic field coupling. By combining the close binary scenario (Hachisu et al. 2012), we have found that the WD evolves as follows, as will be described in detail in Section 3.

(1) For certain ranges of binary parameters, as will be presented in Section 4, the accretion rate $(\dot{M})$ always exceeds $10^{-7} M_{\odot} \mathrm{yr}^{-1}$ so that the WD increases its mass until it undergoes "prompt" carbon ignition. The mass of the uniformly rotating WD at the carbon ignition, $M_{\mathrm{Ig}}^{\mathrm{R}}$, is larger for smaller $\dot{M}$. For $\dot{M}=10^{-7} M_{\odot} \mathrm{yr}^{-1}, M_{\mathrm{Ig}}^{\mathrm{R}}=1.43 M_{\odot}$, which is the largest mass because nova-like hydrogen flashes prevent the the WD mass from growing for the lower $\dot{M}$. Because of 
the centrifugal force in the rotating $\mathrm{WD}, M_{\mathrm{Ig}}^{\mathrm{R}}=1.43 M_{\odot}$ is larger than $M_{\mathrm{Ig}}^{\mathrm{NR}}=1.38 M_{\odot}$ (Nomoto et al. 1984).

(2) For adjacent ranges of binary parameters (Section 4), the mass of the rotating WD exceeds $M_{\mathrm{Ig}}^{\mathrm{NR}}=1.38 M_{\odot}$ but does not reach $M_{\mathrm{Ig}}^{\mathrm{R}}=1.43 M_{\odot}$ because of the decreasing accretion rate. In this Letter, we shall present the evolution of the WD whose mass reaches $1.40 M_{\odot}$, being slightly below $M_{\mathrm{Ig}}^{\mathrm{R}}$.

After the accretion rate falls off, the WD undergoes the $J$-loss evolution. The exact mechanism and the timescale of the $J$-loss are highly uncertain, although we consider the magnetodipole braking WD to be responsible. We have found that $J$-loss induces the contraction of the WD, which leads to the "delayed" carbon ignition after the "delay" time due to neutrino and radiative cooling.

We also studied how the carbon ignition depends on the strong screening factor, which still involves some uncertainties. Then, we shall discuss whether the WD undergoes the SN Ia explosion or collapse (Section 3). Finally, we discuss our findings in Section 4.

\section{METHODS}

We have employed the hydrostatic stellar evolution code described in Benvenuto et al. (2013), adapted to the case of accreting WDs. The equation of state of degenerate electrons is treated as in Kippenhahn \& Thomas (1964), while Coulomb corrections at finite temperature are included following Hansen (1973). Radiative opacities are taken from Iglesias \& Rogers (1996), whereas conductive opacities were included following Itoh et al. (2008). Neutrino emission processes are those described in Itoh et al. (1996).

As stated above, in this Letter, we consider uniform rotation. For each isobar, we computed the parameter $\lambda$, defined as

$$
\lambda=\frac{\Omega^{2} r_{P}^{3}}{2 G M_{P}}
$$

where $\Omega$ is the angular rotation, $r_{P}$ is the radius of a sphere with a volume equal to the corresponding isobar, and $M_{P}$ is the embraced mass. $\lambda$ measures the fastness of rotation; breakup rotation rate corresponds to $\lambda \approx 0.270$.

Let us define the average $\langle q\rangle$ of an attribute $q$ on a given isobar as

$$
\langle q\rangle=\left(\frac{1}{S_{P}}\right) \oint_{P} q d \sigma
$$

where $S_{P}$ is the total surface of the isobar and $d \sigma$ an element of it.

In order to compute the structure of the rotating star, we need the coefficients $f_{P}$ and $f_{T}$ that correct the standard structure equations for non-rotating objects. These are given by

$$
f_{P}=\left(\frac{4 \pi r_{P}^{4}}{G M_{P} S_{P}}\right) \frac{1}{\left\langle g_{\text {eff }}^{-1}\right\rangle}, \quad f_{T}=\left(\frac{4 \pi r_{P}^{2}}{S_{P}}\right)^{2} \frac{1}{\left\langle g_{\text {eff }}^{-1}\right\rangle\left\langle g_{\text {eff }}\right\rangle},
$$

where $g_{\text {eff }}$ is the effective gravitational acceleration, computed in the Roche model approximation (Maeder 2009).

When the WD is accreting, we impose the critical rotation regime for $\lambda=0.270$ for the outermost layers. This provides the (uniform) rotation rate of the entire star.
For the mass accretion rate, we assume that $\dot{M}$ is constant during the main accretion stage and then declines exponentially with an assumed timescale $\tau_{\dot{M}}=4 \times 10^{4}$ years (set in order to mimic binary evolution) since a moment chosen in order for the WD to reach an assumed final mass value $M$. Since $\dot{M} \leqslant 1 \times 10^{-7} M_{\odot} \mathrm{yr}^{-1}$, we also consider $J$-losses with another timescale $\tau_{J}$ so that

$$
J=J\left(t_{0}\right) \exp \left(-\left(t-t_{0}\right) / \tau_{J}\right)
$$

where $t_{0}$ is the age at which $\dot{M}=1 \times 10^{-7} M_{\odot} \mathrm{yr}^{-1}$. We have chosen this prescription to explore the reaction of the WD to $J$-losses without assuming a particular physical process, which at present is uncertain. For this reason, we shall treat $\tau_{J}$ as a free parameter. A possible mechanism leading to such losses is magneto-dipole braking (Ilkov \& Soker 2012; Rueda et al. 2013). For $t>t_{0}$, we compute the rotation rate by considering the instantaneous moment of inertia of the oblate star. Due to $J$-losses, $\lambda$ falls down, although the WD may even rotate faster, leading to internal compression.

For the carbon-burning ${ }^{12} \mathrm{C}+{ }^{12} \mathrm{C}$ reaction rate, we consider the expressions given in Caughlan \& Fowler (1988). A key ingredient is the screening of this reaction. Here, we shall explore four different treatments, which are those of Itoh et al. (1990), Ogata (1997), Kitamura (2000), and Potekhin \& Chabrier (2012). Kitamura (2000) computed the enhancement factors of nuclear reaction rates due to interionic correlations within the linear-mixing (LM) approximation, where polarization of electrons was explicitly taken into account. More recently, Potekhin \& Chabrier (2012) claimed that the deviation $\Delta h$ from the LM approximation tends to reduce the enhancement factor significantly, but their formula (19) is wrong; following their notations, the correct formula should read

$$
\begin{aligned}
& \Delta h \equiv h_{0}-h_{\mathrm{lm}} \\
& =f_{\text {mix }}\left(x_{\mathrm{C}}, x_{\mathrm{O}}, 0\right) \\
& \quad-\left.\frac{d f_{\text {mix }}\left(x_{\mathrm{C}}-\left(2-x_{\mathrm{C}}\right) \xi, x_{\mathrm{O}}(1+\xi), \xi\right)}{d \xi}\right|_{\xi \rightarrow 0},
\end{aligned}
$$

where $f_{\text {mix }}\left(x_{\mathrm{C}}, x_{\mathrm{O}}, x_{\mathrm{Mg}}\right)$ represents the deviation of the interaction free energy from the $\mathrm{LM}$ value for $\mathrm{C}-\mathrm{O}-\mathrm{Mg}$ three-component plasma, with $x_{i}(i=\mathrm{C}, \mathrm{O}, \mathrm{Mg})$ denoting their molar fractions. When this correct formula is adopted, $\Delta h$ turns out negligibly small under the present parameter conditions. For practical use, they also suggested a simple Salpeter-Van Horn-type interpolation formula combined with the quantum correction, but we shall not use it here since it underestimates the reaction rates by one or two orders of magnitude near the freezing point, exhibiting large discrepancies with the pycnonuclear rates in the solid phase (Kitamura 2000). We also note that Itoh et al. (1990) and Ogata (1997) ignored the electron polarization effects. Consequently, we shall consider the treatment by Kitamura (2000) as the standard case and is the one employed unless stated otherwise.

\section{RESULTS}

In this work, we restrict ourselves to the case of $\dot{M}=10^{-6} M_{\odot} \mathrm{yr}^{-1}$; such material is burned in the outermost WD layers to $\mathrm{C}+\mathrm{O}$ composition. The initial composition is $X\left({ }^{12} \mathrm{C}\right)=X\left({ }^{16} \mathrm{O}\right)=0.49$, and heavier elements have solar abundances. 


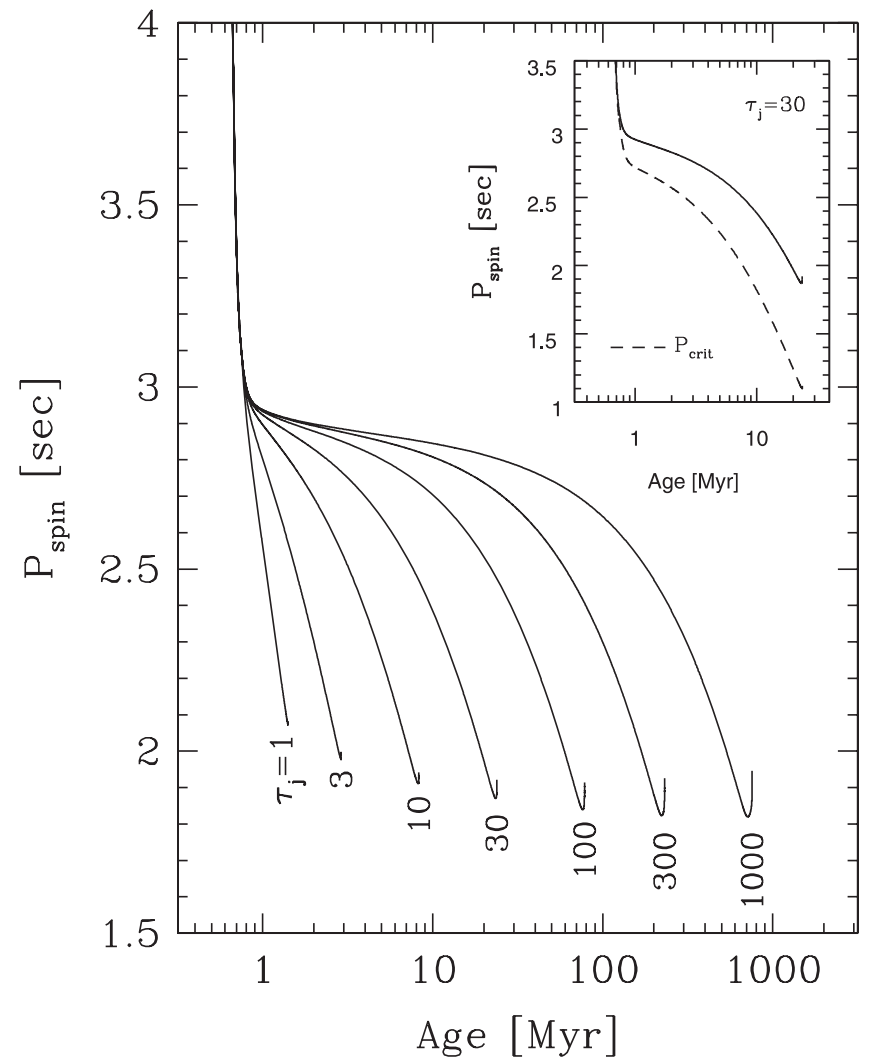

Figure 1. Spin period for the presupernova evolution of a WD for the case of screening of carbon-burning reaction given by Kitamura (2000). The final mass is $1.40 M_{\odot}$. Accretion occurs for $P_{\text {spin }} \gtrsim 2.9 \mathrm{~s}$. Each curve is labeled with its corresponding value of $\tau_{J}$ given in million years. Although the star loses angular momentum exponentially, its moment of inertia decreases fast enough to make the star spin up after the end of accretion. However, remarkably, the star does not overflow its critical rotation rate avoiding shedding mass. This is shown in the inset for the case of $\tau_{J}=30 \mathrm{Myr}$, where spin and critical spin period evolution are given as solid and dashed lines, respectively.

We computed several evolutionary situations that depend on the binary parameters (orbital period $P$-donor mass $M_{2}$ ) as shown in Section 4, stopping calculations before the onset of hydrodynamical stages of thermonuclear runaway (log $\left(T_{\mathrm{c}} / \mathrm{K}\right) \sim 8.8$; Nomoto 1982$)$.

(1) One is the case in which the WD accretes material continuously up to "prompt" carbon ignition. As described in Section 1, we obtained $M_{\mathrm{Ig}}^{\mathrm{R}}=1.43 M_{\odot}$ for $\dot{M}=10^{-7} M_{\odot} \mathrm{yr}^{-1}$. This is smaller than the carbon ignition mass of $1.48 M_{\odot}$ obtained by Uenishi et al. (2003) because their 2D uniformly rotating model neglects the Coulomb term in the equation of state.

(2) We also consider a binary system in which the accretion rate declines and the WD reaches a final mass $M$ before carbon ignition; in this work, we shall consider the case of $M=1.40 M_{\odot}$. For $t>t_{0}$, we apply $J$-losses as given in Equation (4) with timescales of $\tau_{J}=1,3,10,30,100,300$, and $1000 \mathrm{Myr}$. As quoted above, $J$-loss processes are highly uncertain; thus, we employ a wide range for $\tau_{J}$ to find the dependence of WD evolution with this parameter. The main results are presented in Figures 1-4.

In Figure 1, we show the evolution of the spin period $P_{\text {spin }}$ for the presupernova evolution of a WD. Accretion occurs for $P_{\text {spin }} \gtrsim 2.9 \mathrm{~s}$. After the end of accretion, the WD loses angular momentum exponentially (Equation (4)). Despite that, its

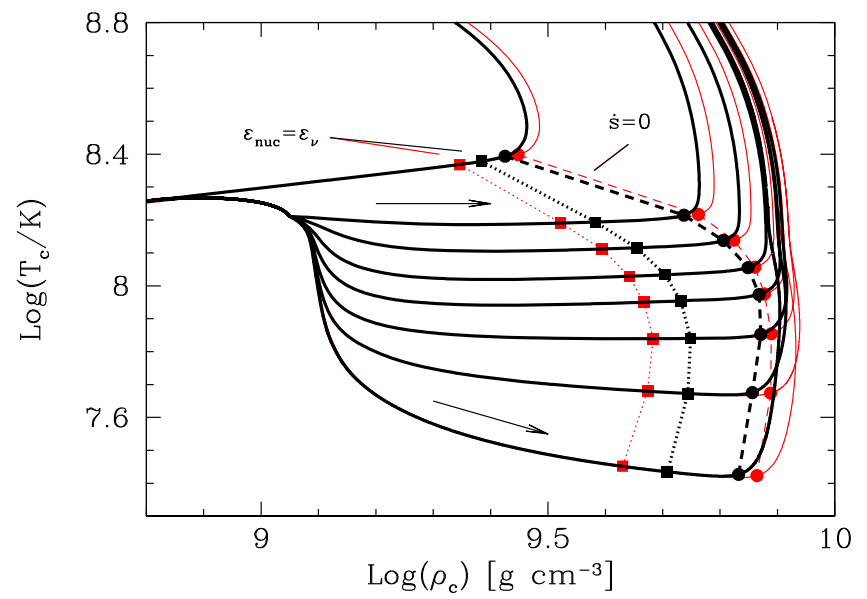

Figure 2. Evolutionary tracks of the center of the WD up to the onset of the hydrodynamical explosion. The upper track corresponds to "prompt" carbon ignition, while the others are ordered from top to bottom with increasing $J$-loss timescale $\left(\tau_{J}=1,3,10,30,100,300\right.$, and $1000 \mathrm{Myr}$, respectively). $\varepsilon_{\mathrm{nuc}}=\varepsilon_{\nu}$ indicates the conditions at which neutrino losses equal nuclear energy release, while $\dot{S}=0$ shows the stages at which central entropy per baryon begins to increase. Thick black and thin red lines correspond to the treatments of screening given by Kitamura (2000) and Potekhin \& Chabrier (2012), respectively. Arrows indicate the sense of the evolution.

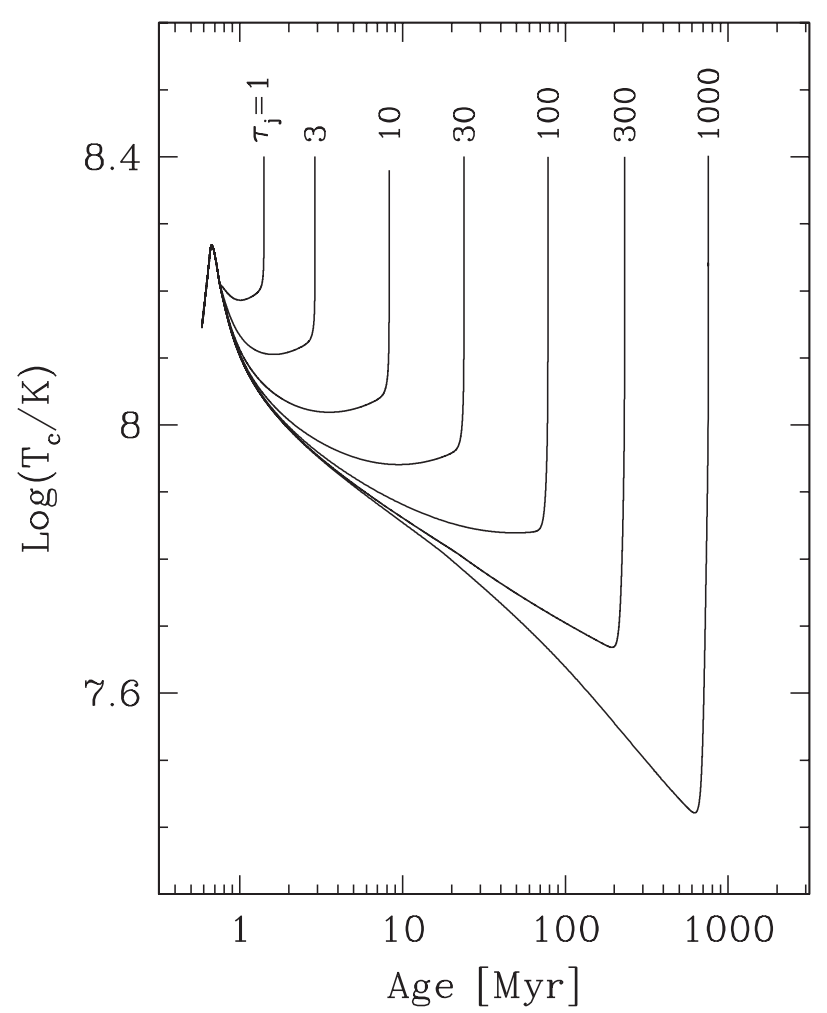

Figure 3. Evolution of the central temperature as a function of time for the cases included in Figure 1.

moment of inertia decreases fast enough for the WD to spin up rather than spin down. We thus do not call such an evolution "spin-down," rather just " $J$-loss" evolution. However, remarkably, the WD does not overflow the critical rotation rate avoiding shedding mass. This is shown in Figure 1 for the case of $\tau_{J}=30$ Myr. This is crucial for the delayed explosion scenario to work because a little mass loss would be enough to preclude the 


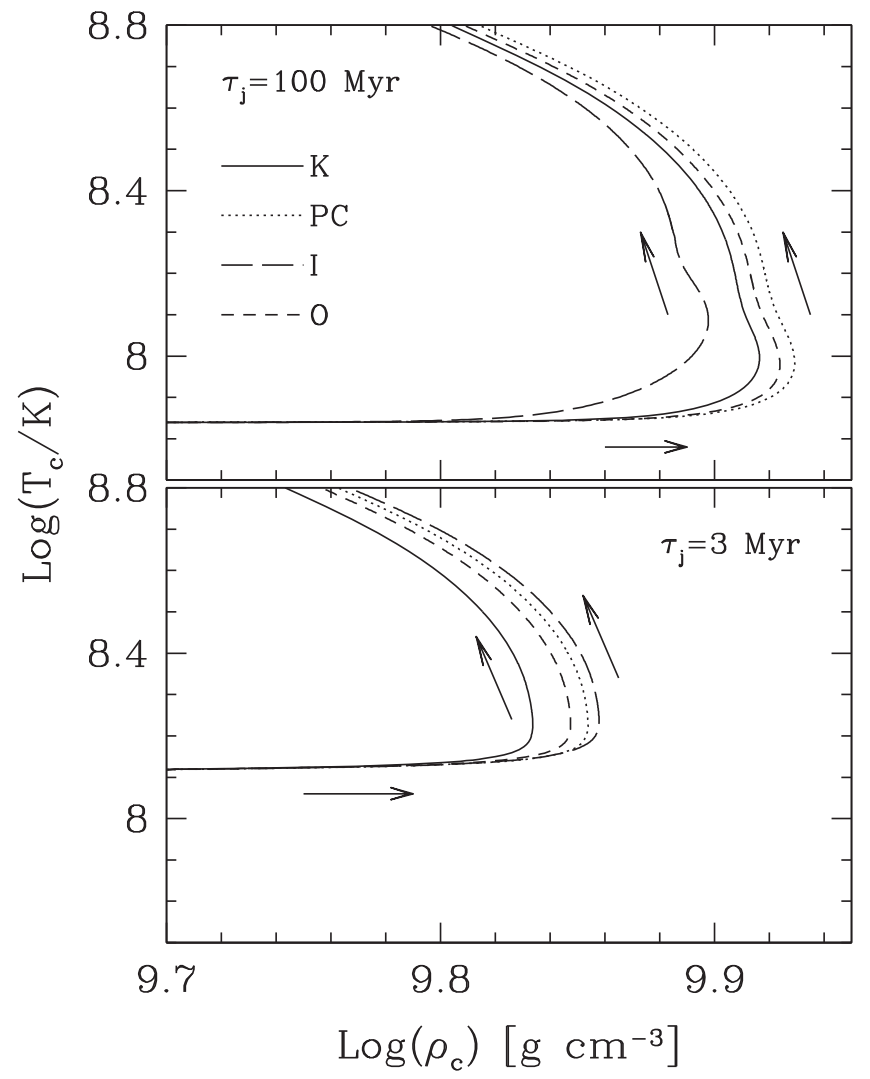

Figure 4. Evolutionary tracks for the center of the WD during carbon ignition for the cases of $\tau_{J}=3$ and $100 \mathrm{Myr}$. I, O, K, and PC correspond to the results employing the treatments for the screening given in Itoh et al. (1990), Ogata (1997), Kitamura (2000), and Potekhin \& Chabrier (2012), respectively. Arrows indicate the sense of the evolution.

occurrence of the explosion. In all cases, $P_{\text {spin }}$ decreases (i.e., spin up rather than spin down) up to a minimum value slightly before explosion; however, $\lambda$ decreases monotonically.

In Figure 2, we present the evolution of the center of the WD since before the end of accretion up to the onset of the hydrodynamical stage. Thick black and thin red lines correspond to the strong screening factors given by Kitamura (2000) and Potekhin \& Chabrier (2012), respectively. The uppermost line corresponds to the case (1) evolution that leads to the "prompt" carbon ignition. Below that, the lines from upper to lower correspond to the evolutions for $\tau_{J}=1,3,10$, $30,100,300$, and $1000 \mathrm{Myr}$, respectively, that lead to the "delayed" carbon ignition.

In Figure 2, we also show the conditions at which neutrino losses equals nuclear energy release $\left(\varepsilon_{\text {nuc }}=\varepsilon_{\nu}\right)$ and those that indicate when the central entropy per baryon begins to increase $\dot{S}=0$, i.e., carbon ignition. These curves are well detached because entropy per baryon starts to increases only when nuclear energy releases exceeds neutrino and conductive losses. Conductive losses are comparatively more relevant the longer the $\tau_{J}$ because neutrino losses are more sensitive to temperature than conductive losses.

In Figure 3, we depict the evolution of the central temperature as a function of time for the cases included in Figure 1. It is seen that for longer $\tau_{J}$, the WD is cooler and takes longer time until the carbon ignition occurs.

In Figure 4, we present the evolutionary tracks for the center of the WD from the carbon ignition for the cases of $\tau_{J}=3$ and $100 \mathrm{Myr}$ for the four strong screening factors (I, O, K, and PC)

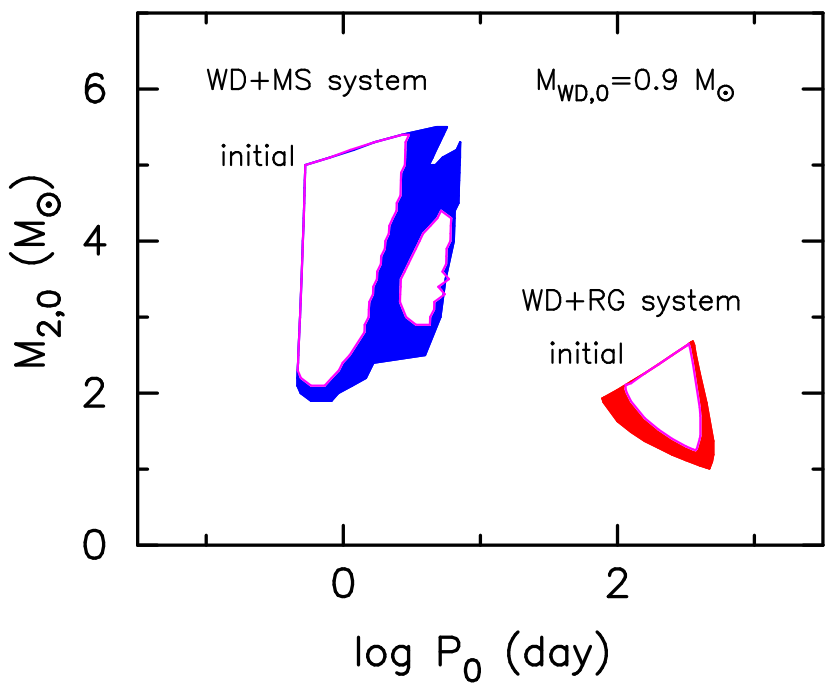

Figure 5. Outcome of the binary evolution of the WD + companion star systems is shown in the parameter space of the initial orbital period $P$ and the companion mass $M_{2}$ for the initial WD mass of $0.9 M_{\odot}$. The mass $M$ of the WD starting from the "painted" region reaches $1.38 M_{\odot}<M<1.43 M_{\odot}$ (delayed carbon ignition), while the systems starting from the blank region encircled by the solid line reach $M=1.43 M_{\odot}$ (prompt carbon ignition).

given in Itoh et al. (1990), Ogata (1997), Kitamura (2000), and Potekhin \& Chabrier (2012), respectively. Note that ignition densities are not so different among the four factors.

If $\tau_{J} \gtrsim 3 \mathrm{Gyr}$, the central temperature, and thus the rate of nuclear energy release, becomes so low that carbon is depleted before reaching the dynamical stage. Due to electron capture, such a case could lead to collapse of the WD rather than explosion, although a detailed study of this phenomenon is necessary (e.g., Förster et al. 2010).

\section{CONCLUSIONS AND DISCUSSION}

The calculations presented above show that a $\mathrm{C}+\mathrm{O}$ WD accreting enough material coming from a binary companion, rotating uniformly at the critical rate, and reaching a mass $M$ fulfilling $M_{\mathrm{ig}}^{\mathrm{NR}}<M<M_{\mathrm{ig}}^{\mathrm{R}}$ (i.e., $1.38 M_{\odot}<M<1.43 M_{\odot}$ ) may indeed undergo delayed explosion. The above delay time is largely determined by the timescale of $J$-losses $\tau_{J}$ (see Figure 3). For the values of $\tau_{J}$ considered here, the WD spends a time to undergo SN Ia explosion enough for the donor star to evolve to a structure completely different from the one it had when acted as a donor. For the red giant donor, its H-rich envelope would be lost as a result of $\mathrm{H}$-shell burning and mass loss so that it would become a He WD in $\sim 10 \mathrm{Myr}$. For the main-sequence donor, it would also evolve to become a lowmass $\mathrm{He}$ WD in $1 \mathrm{Myr}$, a hot $\mathrm{He}$ WD in $10 \mathrm{Myr}$, and a cold $\mathrm{He}$ WD in 1000 Myr (Di Stefano et al. 2011). So, the J-losses should delay the explosion in enough time for the former donor to be undetectable. Therefore, this scenario provides a way to account for the failure in detecting companions to $\mathrm{SNe}$ Ia.

Then, it is important to know for what binary systems the uniformly rotating WD effectively stops increasing its mass $M$ at $1.38 M_{\odot}<M<1.43 M_{\odot}$ and undergoes the delayed carbon ignition. We have calculated the binary evolution of the WD + companion star systems with the parameters of the initial orbital period $P$ and the companion mass $M_{2}$ for the several initial WD masses as in Hachisu et al. (2012). The result for the initial WD mass of $0.9 M_{\odot}$ is shown in Figure 5. 
Here, the binary systems starting from the "painted" region of the $\left(P-M_{2}\right)$ plane reach $1.38 M_{\odot}<M<1.43 M_{\odot}$, while the systems starting from the blank region encircled by the solid line reach $M=1.43 M_{\odot}$. We estimate that the occurrence frequency of the delayed carbon ignition would roughly be one-fourth of the total frequency of the carbon ignition. The occurrence frequency is smaller than the estimate by Wang et al. (2014), possibly because they adopted the mass range of the $J$-loss evolution as $1.38 M_{\odot}<M<1.5 M_{\odot}$.

While here we have restricted ourselves to present results corresponding to the case of WDs that reach a mass of $1.40 M_{\odot}$ with $\dot{M}=10^{-6} M_{\odot} \mathrm{yr}^{-1}$, we have explored this scenario for other final masses and accretion rates. We found that the overall evolution of the objects is qualitatively the same if the final WD mass is close enough to $M_{\mathrm{ig}}^{\mathrm{R}}$. While we did not attempt to determine the lowest-mass value for ignition to occur, we found that models with $1.37 M_{\odot}$ do not ignite, while those with $1.38 M_{\odot}$ explode. This is consistent with the adopted value of $M_{\mathrm{Ig}}^{\mathrm{NR}}$.

We explored different screening theories for the carbonburning reaction, finding that there is an uncertainty in the ignition density of $\approx 10 \%$. Remarkably, ignition (defined as the moment at which entropy per baryon of matter at the center of the star begins to increase) occurs at a density significantly higher than the one at which nuclear energy release equals neutrino losses. This is due to conductive losses, especially important for the case of large $\tau_{J}$ values for which the WD interior is cool.

Carbon burning forms a convective core, which leads to the occurrence of the convective URCA process (e.g., Lesaffre et al. 2005). In the present study, we do not take into account this process, assuming that it does not suppress the heating by carbon burning. After the thermonuclear runaway, the central region would reach nuclear statistical equilibrium and undergo electron capture at higher densities than in the non-rotating WD (Nomoto et al. 1984) as well as the prompt ignition. This would lead to the synthesis of more neutron-rich Fe-peak elements, such as ${ }^{54} \mathrm{Fe},{ }^{56} \mathrm{Fe}$, and ${ }^{58} \mathrm{Ni}$. It would be important to study such nucleosynthesis in multi-dimensional simulation of convective deflagration because the results would be sensitive to the treatment of turbulent flame.

The nucleosynthesis results should be compared with observational signatures of SNe Ia. For example, the emission lines observed in the spectra of the nebular phase of SNe Ia indicate the existence of a large amount of stable Fe and Ni. Mazzali et al. (2007) estimated the amount of stable $\mathrm{Fe}$ is $\sim 0.2 M_{\odot}$ for most normal SNe Ia (see also Mazzali et al. 2015 for SN $2011 \mathrm{fe}$ ). If such an amount of stable Fe is mostly ${ }^{54} \mathrm{Fe}$ and those $\mathrm{SNe}$ Ia are powered by the radioactive decay of $\sim 0.6 M_{\odot}{ }^{56} \mathrm{Ni}$, the ratio of ${ }^{54} \mathrm{Fe} /{ }^{56} \mathrm{Fe}$ in the ejecta of those $\mathrm{SNe}$ Ia is $\sim 8$ times larger than the solar ratio. This suggests that the stable $\mathrm{Fe}$ is mostly ${ }^{56} \mathrm{Fe}$ rather than ${ }^{54} \mathrm{Fe}$, which would be realized for lower $Y_{e}$, i.e., at the higher central density than the prompt ignition model (such as W7 in Nomoto et al. 1984). Lower $Y_{e}$ in the delayed carbon burning might also be consistent with the large amount of $\mathrm{Mn}$ and (stable) $\mathrm{Ni}$ observed in the SN Ia remnants (Yamaguchi et al. 2015).

We would like to thank N. Itoh and S. Ogata for useful comments on the strong screening factor. This work has been supported in part by the WPI Initiative, MEXT, Japan, and by the Grants-in-Aid for Scientific Research of the JSPS (23224004, 24540227, and 26400222).

\section{REFERENCES}

Benvenuto, O. G., Bersten, M. C., \& Nomoto, K. 2013, ApJ, 762, 74 Caughlan, G. R., \& Fowler, W. A. 1988, ADNDT, 40, 283

Dilday, B., Howell, D. A., Cenko, S. B., et al. 2012, Sci, 337, 942

Di Stefano, R., Voss, R., \& Claeys, J. S. W. 2011, ApJL, 738, LL1

Foley, R. J., Simon, J. D., Burns, C. R., et al. 2012, ApJ, 752, 101

Förster, F., Lesaffre, P., \& Podsiadlowski, P. 2010, ApJS, 190, 334

Hachisu, I., Kato, M., \& Nomoto, K. 2012, ApJL, 756, LL4

Hamuy, M., Phillips, M. M., Suntzeff, N. B., et al. 2003, Natur, 424, 651

Hansen, J. P. 1973, PhRvA, 8, 3096

Hillebrandt, W., \& Niemeyer, J. C. 2000, ARA\&A, 38, 191

Iglesias, C. A., \& Rogers, F. J. 1996, ApJ, 464, 943

Ilkov, M., \& Soker, N. 2012, MNRAS, 419, 1695

Itoh, N., Hayashi, H., Nishikawa, A., \& Kohyama, Y. 1996, ApJS, 102, 411

Itoh, N., Kuwashima, F., \& Munakata, H. 1990, ApJ, 362, 620

Itoh, N., Uchida, S., Sakamoto, Y., Kohyama, Y., \& Nozawa, S. 2008, ApJ, 677,495

Justham, S. 2011, ApJL, 730, LL34

Kerzendorf, W. E., Schmidt, B. P., Asplund, M., et al. 2009, ApJ, 701, 1665

Kerzendorf, W. E., Schmidt, B. P., Laird, J. B., Podsiadlowski, P., \& Bessell, M. S. 2012, ApJ, 759, 7

Kippenhahn, R., \& Thomas, H.-C. 1964, ZA, 60, 19

Kitamura, H. 2000, ApJ, 539, 888

Lesaffre, P., Podsiadlowski, P., \& Tout, C. A. 2005, MNRAS, 356, 131

Li, W., Bloom, J. S., Podsiadlowski, P., et al. 2011, Natur, 480, 348

Maeder, A. 2009, Physics, Formation and Evolution of Rotating Stars (Berlin: Springer)

Maoz, D., Mannucci, F., \& Nelemans, G. 2014, ARA\&A, 52, 107

Mazzali, P. A., Röpke, F. K., Benetti, S., \& Hillebrandt, W. 2007, Sci, 315, 825

Mazzali, P. A., Sullivan, M., Filippenko, A. V., et al. 2015, MNRAS, 450,2631

Nomoto, K. 1982, ApJ, 257, 780

Nomoto, K., Thielemann, F.-K., \& Yokoi, K. 1984, ApJ, 286, 644

Nomoto, K., Umeda, H., Kobayashi, C., et al. 2000, in AIP Conf. Ser., 522, 35

Ogata, S. 1997, ApJ, 481, 883

Ostriker, J. P., \& Bodenheimer, P. 1968, ApJ, 151, 1089

Patat, F., Chandra, P., Chevalier, R., et al. 2007, Sci, 317, 924

Potekhin, A. Y., \& Chabrier, G. 2012, A\&A, 538, AA115

Rueda, J. A., Boshkayev, K., Izzo, L., et al. 2013, ApJL, 772, L24

Schaefer, B. E., \& Pagnotta, A. 2012, Natur, 481, 164

Sternberg, A., Gal-Yam, A., Simon, J. D., et al. 2011, Sci, 333, 856

Uenishi, T., Nomoto, K., \& Hachisu, I. 2003, ApJ, 595, 1094

Wang, B., Justham, S., Liu, Z.-W., et al. 2014, MNRAS, 445, 2340

Yamaguchi, H., Badenes, C., Foster, A. R., et al. 2015, ApJL, 801, L31

Yoon, S.-C., \& Langer, N. 2005, A\&A, 435, 967 